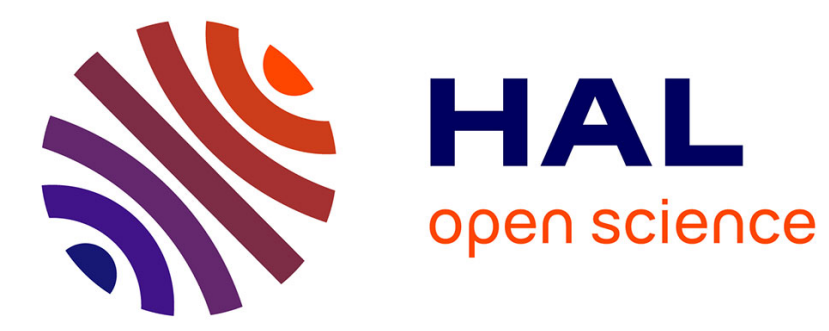

\title{
Étude de plasmas de synthèse pour la simulation du vol ionosphérique
}

\author{
G. Fournier, D. Pigache
}

\section{To cite this version:}

G. Fournier, D. Pigache. Étude de plasmas de synthèse pour la simulation du vol ionosphérique. Revue de Physique Appliquée, 1977, 12 (7), pp.1063-1070. 10.1051/rphysap:019770012070106300 . jpa-00244279

\section{HAL Id: jpa-00244279 https://hal.science/jpa-00244279}

Submitted on 1 Jan 1977

HAL is a multi-disciplinary open access archive for the deposit and dissemination of scientific research documents, whether they are published or not. The documents may come from teaching and research institutions in France or abroad, or from public or private research centers.
L'archive ouverte pluridisciplinaire HAL, est destinée au dépôt et à la diffusion de documents scientifiques de niveau recherche, publiés ou non, émanant des établissements d'enseignement et de recherche français ou étrangers, des laboratoires publics ou privés. 


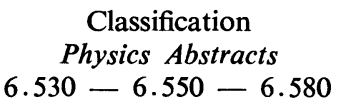

\title{
ÉTUDE DE PLASMAS DE SYNTHËSE POUR LA SIMULATION DU VOL IONOSPHÉRIQUE (1)
}

\author{
G. FOURNIER et D. PIGACHE
}

Office National d'Etudes et de Recherches Aérospatiales (O. N. E. R. A.), 92320 Châtillon, France

(Reçu le 7 février 1977, accepté le 23 mars 1977)

\begin{abstract}
Résumé. - La simulation du vol d'un satellite dans l'ionosphère a été obtenue dans une grande chambre à vide au moyen d'un plasma de synthès z sans collision. La densité de plasma, la température électronique et le nombre de Mach électronique peuvent varier entre $10^{4}$ et $10^{6} \mathrm{~cm}^{-3}, 500$ et $2000 \mathrm{~K}, 8$ et 40 respectivement. Les deux principales difficultés ont été : la mise au point d'une source d'ions de forte intensité $(50 \mathrm{~mA})$ et d'énergie très basse $(20 \mathrm{eV})$ et l'obtention de températures électroniques exceptionnellement basses pour un plasma de laboratoire. Une étude quantitative du sillage de corps sphériques et cylindriques a été effectuée grâce à de nouveaux perfectionnements : la mesure et le réglage de la température des ions et l'abaissement de la pression résiduelle (source de plasma en fonctionnement) de $10^{-4} \mathrm{~N} / \mathrm{m}^{2}$ à $1,5 \times 10^{-5} \mathrm{~N} / \mathrm{m}^{2}$.
\end{abstract}

\begin{abstract}
The simulation of satellite flight in the ionosphere has been obtained in a large vacuum chamber by means of a collisionless synthetized plasma. The plasma density, the electron temperature and the electronic Mach number can be varied between $10^{4}$ and $10^{6} \mathrm{~cm}^{-3}, 500$ and $2000 \mathrm{~K}, 8$ and 40 respectively. The two principal difficulties have been the development of a high intensity $(50 \mathrm{~mA})$ and low energy $(20 \mathrm{eV})$ ion source and the obtaining of unusually low electron temperature for a laboratory plasma. A quantitative study of the wake of spherical and cylindrical bodies has been performed owing to new improvements : measurement and adjustment of the ion temperature and reducing the residual pressure (plasma source working) from $10^{-4} \mathrm{~N} / \mathrm{m}^{2}$ to $1.5 \times 10^{-5} \mathrm{~N} / \mathrm{m}^{2}$.
\end{abstract}

1. Introduction. - Un plasma de synthèse est obtenu par injection d'électrons dans un faisceau d'ions positifs. Les premières études de ce type de plasma remontent à la mise en évidence en laboratoire de la possibilité de neutraliser en courant et en charge les faisceaux d'ions intenses prévus pour la propulsion spatiale (moteur ionique). Les principales propriétés de ces plasmas ont été étudiées dans des enceintes à vide poussé et en régime impulsionnel afin de se rapprocher de l'ambiance spatiale et d'éviter le phénomène d'autoneutralisation du faisceau par les électrons résultant de l'ionisation du gaz par le faisceau [1], [2], [3].

La simulation de l'environnement ionosphérique des satellites peut être obtenue au moyen d'un plasma de synthèse dirigé vers une maquette. Un certain nombre de souffleries à plasma fonctionnant sur ce principe ont été construites ces dernières années, par exemple [4], [5], [6], [7].

La plupart d'entre elles n'ont été utilisées que pour des recherches fondamentales telles que l'étude du sillage d'un satellite ou l'analyse des propriétés des plasmas en mouvement. Les lois de similitude sont

(1) Communication présentée au Congrès National de Physique des Plasmas, Paris, 6-10 décembre 1976. fréquemment employées pour ces recherches fondamentales et la plupart des conditions expérimentales sont alors relativement faciles à obtenir.

Cependant la calibration en laboratoire des instruments de diagnostic de plasma qui vont être utilisés à bord d'un véhicule spatial nécessite un régime de fonctionnement continu et ne permet aucun changement d'échelle. Ces instruments (et le volume de plasma analysé) sont généralement de grande taille et les circuits électroniques associés sont adaptés aux conditions ionosphériques qui doivent être reproduites exactement au laboratoire (en particulier la fréquence plasma, la gyrofréquence électronique et la température électronique). La simulation exacte des effets de sillage sur ces instruments exige aussi que la source d'ions fonctionne avec une tension accélératrice très basse. (En effet un paramètre de similitude essentiel à considérer est le nombre de Mach électronique

$$
M \simeq V\left(k T_{\mathrm{e}} / m\right)^{-1 / 2}=\left(2 E / k T_{\mathrm{e}}\right)^{1 / 2}
$$

où $V$ est la vitesse dirigée des ions, $k$ la constante de Boltzman, $T_{\mathrm{e}}$ la température électronique, $m$ la masse des ions et $E$ l'énergie des ions).

L'emploi d'un faisceau d'ions d'énergie très basse a en outre l'avantage de réduire à un niveau négligeable 
la perturbation due aux électrons produits par émission secondaire sur les obstacles.

Pour ces raisons, une soufflerie à plasma de grandes dimensions a été réalisée avec un effort particulier pour obtenir autant que possible une simulation exacte du plasma ionosphérique dans un grand volume.

Les principales difficultés à résoudre ont été :

- la mise au point d'une source d'ions de forte intensité (quelques dizaines de $\mathrm{mA}$ ) avec une tension accélératrice très basse $(\simeq 20 \mathrm{~V})$;

- l'obtention et la mesure de températures électroniques égales à celles rencontrées dans l'ionosphère $(500$ à $2000 \mathrm{~K})$ c'est-à-dire exceptionnellement basses pour un plasma de laboratoire ;

- l'obtention et la mesure de températures ioniques du même ordre de grandeur $\left(T_{\mathrm{i}} \lesssim T_{\mathrm{e}}\right)$;

- l'élimination de toute perturbation due aux molécules neutres nécessitant un vide poussé avec la source de plasma en fonctionnement.

2. Description du montage et méthodes de mesure. Le plasma de synthèse est créé dans un grand caisson à vide (dimensions intérieures $3 \mathrm{~m}$ de diamètre et $5 \mathrm{~m}$ de longueur). La pression résiduelle normale avec la source de plasma en fonctionnement est égale à $10^{-4} \mathrm{~N} / \mathrm{m}^{2}\left(1 \mathrm{~N} / \mathrm{m}^{2}=0,75 \times 10^{-2}\right.$ torr $)$. La figure 1 montre le schéma de l'installation. La source de plasma

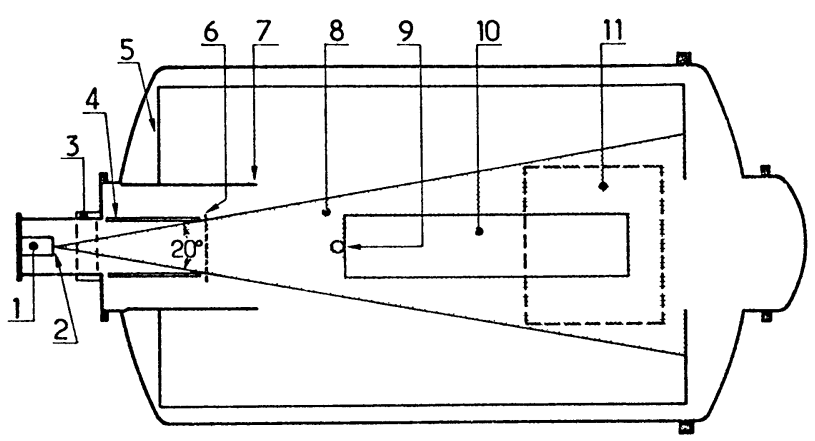

FIG. 1. - Schéma du dispositif expérimental. 1) Source d'ions. 2) Ouverture de la source d'ions. 3) Vanne $(\varnothing=50 \mathrm{~cm})$. 4) Piège à hélium liquide. 5) Ecran en acier inoxydable avec revêtement intérieur isolant $(\varnothing=3 \mathrm{~m} ; 1=5 \mathrm{~m}) .6$ ) Grille pour la simulation de Ti. 7) Ecran en aluminium poli. 8) Faisceau d'ions. 9) Obstacle sphérique ou cylindrique. 10) Zone explorée dans les études de sillage. 11) Zone utilisée pour les essais d'expériences spatiales.

(décrite aux $\S \S 3$ et 4 ) est placée à une extrémité de la chambre et peut être isolée de celle-ci au moyen d'une vanne de $50 \mathrm{~cm}$ de diamètre. La zone utilisée pour les études de sillage est située au centre du caisson. Celle utilisée pour les essais d'expériences spatiales est située à l'opposé de la source d'ions; le diamètre utile du plasma est de l'ordre de $2 \mathrm{~m}$ et la variation axiale de la densité est réduite au minimum (variation en $1 / r^{2}$ en partant de la source, $r \simeq 5 \mathrm{~m}$ ).

Les méthodes de mesure employées pour caractériser le plasma sont les suivantes :
- sondes de Langmuir pour la densité de plasma et la température électronique ;

- analyseur électrostatique et mesure de temps de vol pour la vitesse dirigée des ions;

- analyseur électrostatique pour la composante longitudinale (parallèle au faisceau) de la température ionique ;

- sonde cylindrique longue et collimateur pour la composante transversale de la température ionique (ces méthodes sont décrites au $\S 5$ ).

Les instruments de mesure peuvent être déplacés (par télécommande sous vide) dans tout le volume du caisson. Les mesures de densité et de température sont en bon accord avec les résultats obtenus avec des sondes hautes fréquences (sonde quadrupolaire et sonde à impédance) par les laboratoires préparant des expériences spatiales [8].

Les mesures de sondes de Langmuir dans un plasma très dilué ont soulevé des difficultés particulières en raison de la contamination des surfaces de sondes qui se traduit par la formation de couches résistives, responsables de chutes de tension de l'ordre de plusieurs volts (plusieurs dizaines de volts dans certains cas). Les caractéristiques de sondes relevées dans ces conditions sont évidemment totalement inexploitables et d'ailleurs non reproductibles même en ce qui concerne le courant de saturation ionique (la sonde polarisée négativement reçoit toujours un courant d'électrons plus ou moins important). En absence de couche résistive, les variations du potentiel de surface de la sonde dues à la contamination introduisent encore des erreurs de l'ordre de quelques centaines de millivolts. Il est alors possible de mesurer correctement la densité des ions mais les erreurs introduites dans la mesure de la température électronique sont importantes et peuvent aisément passer inaperçues : caractéristiques de sondes souvent reproductibles, distribution d'énergie apparemment maxwellienne mais température mesurée deux ou trois fois supéricure à la température réelle.

Aucun nettoyage chimique ne permet d'éliminer ce phénomène. La seule solution consiste à nettoyer la sonde sous vide par bombardement ionique (sonde immergée dans le plasma et polarisée à $-2 \mathrm{kV}$ pendant 20 minutes) ou par bombardement électronique. Même dans l'enceinte à vide poussé et relativement propre utilisée dans ces expériences (pompes à diffusion avec pièges à azote liquide) le temps de recontamination de la sonde est de l'ordre de quelques heures dans le meilleur des cas. Les températures électroniques très basses obtenues dans ces expériences (voir § 4) n'ont été observées qu'après analyse de ce phénomène et mise au point d'une procédure de décontamination efficace.

3. La source d'ions. - Le gaz utilisé le plus souvent est l'argon. Pratiquement la seule espèce d'ions produite est $\mathrm{Ar}^{+}$, de masse voisine de la masse moyenne des constituants de l'ionosphère. Le choix de vapeurs 
métalliques ( $\mathrm{Hg}$ ou Cs) qui aurait grandement facilité l'obtention de vide poussé par pompage cryogénique n'a pas été retenu afin d'éviter la contamination des appareillages d'expériences spatiales.

La densité de plasma à $5 \mathrm{~m}$ de la source atteint $2 \times 10^{6} \mathrm{~cm}^{-3}$, c'est-à-dire la densité maximum susceptible d'être observée dans l'ionosphère et ceci pour un diamètre utile de $2 \mathrm{~m}$. Le courant d'ions correspondant est égal à $50 \mathrm{~mA}$. Le diamètre de la grille d'extraction est égal à $3 \mathrm{~cm}$. La valeur adoptée le plus souvent pour l'énergie des ions est $20 \mathrm{eV}$ ce qui correspond à $10 \mathrm{~km} / \mathrm{s}$ (du même ordre de grandeur que celle d'un satellite) et à un nombre de Mach électronique minimum égal à 8 .

La mise au point de la source d'ions a soulevé quelques difficultés. La chambre d'ionisation est une décharge reflex compacte (longueur et diamètre $10 \mathrm{~cm}$ ) et à cathode chaude assez semblable aux sources Kaufman utilisées pour la propulsion ionique [10] (Fig. 2). Des densités de courant de $4 \mathrm{~mA} / \mathrm{cm}^{2}$ [9] puis

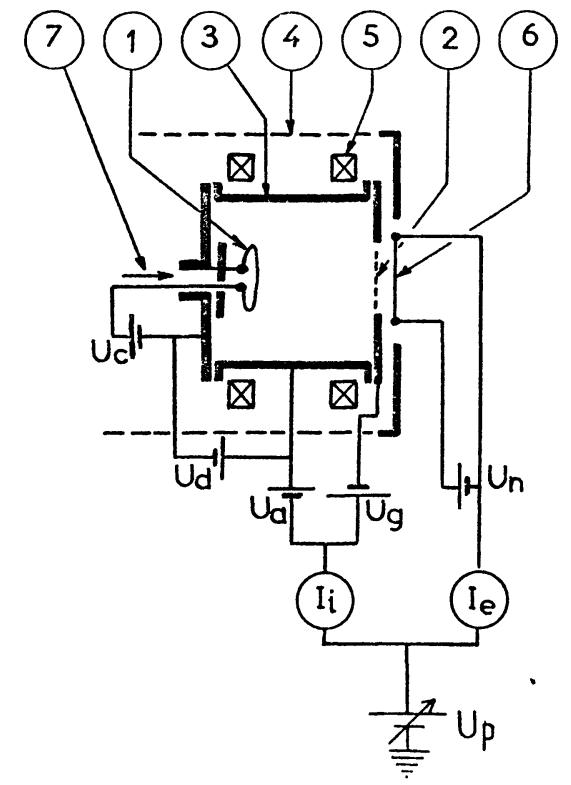

Fig. 2. - Schéma de principe de la source d'ions et de son circuit électrique. 1) Filament de cathode. 2) Grille d'extraction. 3) Anode. 4) Ecran. 5) Bobines magnétiques. 6) Filament neutraliseur. 7) Injection de gaz (argon). $U_{\mathrm{d}}=$ tension de décharge $; U_{\mathrm{a}}^{\prime}=$ tension d'anode $\left(E \simeq e U_{\mathrm{a}}\right) ; U_{\mathrm{g}}=$ tension de grille $; U_{\mathrm{c}}=$ tension de chauffage de la cathode ; $U_{\mathrm{n}}=$ tension de chauffage du neutraliseur; $U_{\mathrm{p}}=$ tension de polarisation de la source ; $I_{\mathrm{i}}=$ courant d'ions émis par la source $; I_{\mathrm{e}}=$ courant d'électrons émis par le neutraliseur.

de $7 \mathrm{~mA} / \mathrm{cm}^{2}$ [10] ont été atteintes. Ce résultat a été obtenu grâce à l'emploi d'une grille d'extraction unique, polarisée négativement, à maille très fine $(100$ à $200 \mu \mathrm{m})$ et de grande transparence $(70 \%)$. Il a été vérifié [10] que la densité de courant maximum susceptible d'être extraite par cette méthode correspond à une épaisseur de gaine au niveau de la grille d'extraction approximativement égale à la dimension des trous.
La densité de plasma ainsi obtenue est suffisante pour les essais d'expériences spatiales. Par contre, pour les études de sillage une densité encore plus élevée serait souhaitable. En effet, l'influence de la divergence du faisceau dans la zone d'interaction doit pouvoir être négligée; dans ces conditions la valeur maximum atteinte pour le rapport $R / h$ ( $R$ rayon du corps sphérique ou cylindrique et $h$ longueur de Debye) est égale à 10 .

Afin d'augmenter le rapport $R / h$ la densité de plasma dans la chambre d'ionisation a été accrue. Il en a résulté un courant d'ions de $800 \mathrm{~mA}$ à $60 \mathrm{eV}$ et de $250 \mathrm{~mA}$ à $20 \mathrm{eV}$. Malheureusement il s'est avéré impossible de neutraliser efficacement un faisceau aussi intense à ces énergies par la technique du filament neutraliseur (décrite au § 4). Plusieurs configurations de neutraliseur et plusieurs diamètres de grille d'extraction (jusqu'à $7 \mathrm{~cm}$ ) ont été essayés. Dans les conditions les plus favorables le courant d'ions utile maximum est de l'ordre de $50 \mathrm{~mA}$ (pour des courants supérieurs la divergence du faisceau augmente de telle sorte que la densité de plasma en un point de l'axe du faisceau reste pratiquement constante).

4. Neutralisation du faisceau d'ions et température électronique. - 4.1 PRINCIPE DE LA NEUTRALISATION. - Le faisceau d'ions est neutralisé par les électrons émis par un filament en tantale chauffé placé à environ $1 \mathrm{~cm}$ de la grille d'extraction. La chute de tension aux bornes du filament est de l'ordre de quelques volts.

Bien que la source d'ions fonctionne en régime continu le phénomène d'autoneutralisation observé par d'autres [1], [2], [3], ne se produit pas même si la pression est augmentée de plus d'un facteur 100 $\left(4 \times 10^{-2} \mathrm{~N} / \mathrm{cm}^{2}\right)$. Ce phénomène d'autoneutralisation dû au piégeage des électrons résultant de l'ionisation du gaz résiduel et de l'émission secondaire des parois de l'enceinte ne peut se produire en raison de l'énergie trop faible des ions ( $E \leqslant 60$ volts). En pratique on constate que sans émission électronique (filament froid) la densité du faisceau baisse d'au moins deux ordres de grandeur et la charge d'espace devient positive.

4.2 OBTENTION DE TEMPÉRATURES ÉLECTRONIQUES BASSES. - Les mesures de sondes de Langmuir montrent que la distribution des énergies des électrons est maxwellienne et que leur température est très basse (typiquement $500 \mathrm{~K}$ ) et pratiquement constante dans tout le plasma. Des températures électroniques aussi basses ont été observées pour la première fois avec une source de plasma de même type dans un petit caisson [9], [12]. Le potentiel du plasma est également constant (à $k T_{\mathrm{e}} / e$ près, $e$ étant la charge de l'électron) et proche du potentiel de la borne négative du neutraliseur (soit $U_{\mathrm{p}}$ voir figure 2). La température du filament, la chute de tension due au chauffage direct et la gaine d'électrons entourant le filament sont telles que l'énergie moyenne des électrons issus du filament est supérieure d'au 
moins un ordre de grandeur à l'énergie des électrons du plasma. Ce fait nécessite une explication.

Environ $95 \%$ de la surface intérieure du caisson est couverte d'un matériau isolant ; cette surface doit être au potentiel flottant par rapport au plasma ; de plus la cible métallique et les sondes sont également flottantes ou polarisées négativement. Dans ces conditions, les électrons subissent un confinement électrostatique partiel. Les électrons d'énergie élevée sont capables de remonter la barrière de potentiel et sont neutralisés sur la paroi tandis que les électrons de basse énergie sont réfléchis. En régime permanent, les électrons perdus sont remplacés par un nombre égal d'électrons émis par le neutraliseur. Le bilan énergétique est négatif et la température électronique doit décroître s'il existe un processus de thermalisation. Cette explication qualitative est confirmée par le fait qu'une distribution maxwellienne et une température basse ne sont observées que si la thermalisation collisionnelle des électrons est possible.

Le courant d'ions $I_{i}$ émis par la source d'ions est pratiquement égal au courant d'électrons $I_{\mathrm{e}}$ émis par le neutraliseur (et ceci quelle que soit la valeur de $U_{\mathrm{p}}$ dans le grand caisson à paroi isolante). Comme les densités électroniques et ioniques sont partout égales entre elles, le temps de confinement moyen $\tau_{\mathrm{e}}$ des électrons est égal à la durée de vie moyenne des ions égale au temps de vol soit $\tau_{\mathrm{e}}=\tau_{\mathrm{i}}=600 \mu \mathrm{s}$ pour $V=10 \mathrm{~km} / \mathrm{s}$ (ions argon de $20 \mathrm{eV}$ ) et un parcours de $6 \mathrm{~m}$.

Le nombre de collisions moyen par électron peut être calculé grâce aux approximations suivantes :

- la densité de plasma est constante dans une couche d'épaisseur $\mathrm{d} r$ perpendiculaire à l'axe du faisceau à la distance $r$ de l'origine virtuelle du faisceau ;

- le flux d'ions est indépendant de $r$ et est égal à $I_{\mathrm{i}} / e$;

- la densité de plasma dans la couche située à la distance $r$ est $n=n_{0} r_{0}^{2} / r^{2}$ où $r_{0}$ est la distance entre l'origine virtuelle du faisceau et la grille d'extraction et $n_{0}$ la densité au niveau de la grille d'extraction avec $n_{0}=I_{\mathrm{i}} / e S V$ où $S$ est la surface de la grille d'extraction ;

- les électrons peuvent se déplacer librement dans tout le faisceau.

La fréquence de collision $v_{\mathrm{e}}$ est proportionnelle à la densité de plasma $v_{\mathrm{e}}=$ an avec $a=3,7 \log \Lambda T_{\mathrm{e}}^{-3 / 2}$ et $\log \Lambda \simeq 10$ [11]. Le nombre d'électrons dans une couche d'épaisseur $\mathrm{d} r$ étant indépendant de $r$ la probabilité de collision d'un électron dans une couche est $\nu_{\mathrm{e}} \tau_{\mathrm{e}} \mathrm{d} r /\left(r_{1}-r_{0}\right)$ où $r_{1}$ est la distance entre l'origine virtuelle du faisceau et la paroi opposée de la chambre. Le nombre de collisions moyen d'un électron est égal à

$$
\begin{aligned}
& \bar{v}_{\mathrm{e}} \tau_{\mathrm{e}}=\frac{a n_{0} r_{0}^{2}}{r_{1}-r_{0}} \tau_{\mathrm{e}} \int_{r_{0}}^{r_{1}} \mathrm{~d} r / r^{2} \\
& \bar{v}_{\mathrm{e}} \tau_{\mathrm{e}}=v_{\mathrm{e} 0} \frac{r_{0}}{r_{1}} \tau_{\mathrm{e}}=v_{\mathrm{e} 1} \frac{r_{1}}{r_{0}} \tau_{\mathrm{e}}
\end{aligned}
$$

où $v_{\mathrm{e} 0}$ et $v_{\mathrm{e} 1}$ sont respectivement les fréquences de coll1sion au niveau de la grille d'extraction et de la paroi opposée de la chambre. Dans des conditions expérimentales typiques $I_{\mathrm{i}}=20 \mathrm{~mA}, S=7 \mathrm{~cm}^{2}, V=10 \mathrm{~km} / \mathrm{s}$, $r_{0}=4 \mathrm{~cm}$ (valeur déduite de la variation de $n(r)$ sur l'axe du faisceau) $n_{0}=1,78 \times 10^{10} \mathrm{~cm}^{-3}$, $n_{1}=0,76 \times 10^{6} \mathrm{~cm}^{-3}$ on obtient pour le nombre de collisions moyen par électron $\bar{v}_{\mathrm{e}} \tau_{\mathrm{e}}=235$. Ce nombre est donc très supérieur à 1 pour les densités de plasma les plus élevées. Des distributions maxwelliennes et des températures basses (comprises entre 500 et $1000 \mathrm{~K}$ ) sont effectivement observées pour toutes les valeurs de densité $n_{1} \approx 10^{4} \mathrm{~cm}^{-3}$; dans ces conditions la relation $\bar{v}_{\mathrm{e}} \tau_{\mathrm{e}}>1$ est toujours respectée. Pour $n_{1} \approx 10^{4} \mathrm{~cm}^{-3}$ la distribution n'est plus maxwellienne et les températures correspondantes, déterminées par la pente moyenne du tracé semi-logarithmique de la caractéristique de sonde, sont de l'ordre de 2000 à $4000 \mathrm{~K}$; dans ces conditions les électrons subissent en moyenne moins d'une collision.

4.3 CONFINEMENT ÉLECTROSTATIQUE DES ÉLECTRONS DANS UNE CHAMBRE A PAROIS MÉTALLIQUES. - Ce résultat a été confirmé par des expériences complémentaires effectuées dans un petit caisson à parois entièrement métalliques avec une source d'ions de même type [9], [12]. Contrairement à ce qui se passe dans un caisson à parois intérieures isolantes dans lequel on a toujours $I_{\mathrm{e}} \simeq I_{\mathrm{i}}$ et $\tau_{\mathrm{e}} \simeq \tau_{1}$, la variation du potentiel du plasma (pratiquement égal à $U_{\mathrm{p}}$, voir figure 2) modifie le degré de confinement électrostatique des électrons. La figure 3 montre la variation des courants $I_{\mathrm{i}}$ et $I_{\mathrm{e}}$ en fonction de $U_{\mathrm{p}}$. On remarque que $I_{\mathrm{i}}$ est pratiquement constant alors que $I_{\mathrm{e}}$ décroît d'un facteur 100 . La durée de vie des ions (ou temps de vol) est égale à $100 \mu \mathrm{s}\left(r_{1}=1 \mathrm{~m}\right.$ et $V=10 \mathrm{~km} / \mathrm{s})$. Le temps de confinement des électrons est $\tau_{\mathrm{e}}=\tau_{\mathrm{i}} I_{\mathrm{i}} / I_{\mathrm{e}}$ et peut donc varier entre $10 \mu \mathrm{s}$ et $1 \mathrm{~ms}$. Le confinement des électrons n'est pas parfait même aux potentiels positifs $U_{\mathrm{p}}$ les plus élevés : $I_{\mathrm{e}} \simeq 60 \mu \mathrm{A}$ pour $U_{\mathrm{p}}=100$ volts. Pour $U_{\mathrm{p}} \sim 100$ volts le plasma est instable (variations brusques de son potentiel).

On remarque que le confinement électrostatique des électrons n'est pas parfait. Le courant résiduel de $60 \mu \mathrm{A}$ est très supérieur au courant d'électrons collecté par les sondes et par les matériaux volants (supports de sondes) immergés dans le faisceau. D'autre part la variation de $U_{\mathrm{p}}$ nécessaire pour modifier ce confinement devrait être de l'ordre de $k T_{\mathrm{e}} / e$; elle est en fait supérieure de plus d'un ordre de grandeur. Ce fait s'explique par la chute de tension à travers la couche résistive due à la contamination des parois de la chambre. La chute de tension à la surface d'une sonde de Langmuir mal nettoyée peut atteindre plusieurs dizaines de volts et l'enceinte ne peut être nettoyée par la méthode la plus efficace décrite au $\S 2$. Il convient de noter que la variation de $I_{\mathrm{e}}$ d'un facteur 100 (Fig. 3) n'a été obtenue qu'après un nettoyage soigné des parois de l'enceinte (solvants usuels). Préalable- 


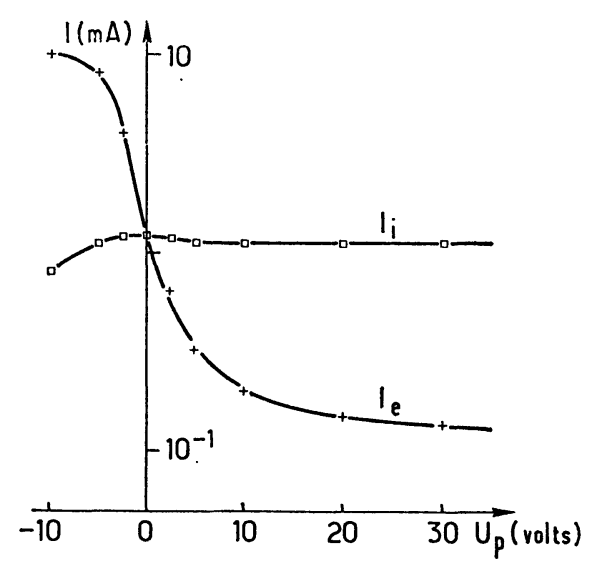

Fig. 3. - Courant d'ions émis par la source $\left(I_{i}\right)$ et courant d'électrons émis par le neutraliseur $\left(I_{\mathrm{e}}\right)$ en fonction de la tension de polarisation de la source $\left(U_{\mathrm{p}}\right)$ dans une enceinte à paroi intérieure conductrice.

ment à ce nettoyage le film de produits isolants (huile de pompe à diffusion principalement) qui recouvrait les parois de l'enceinte était assez résistif pour que l'on ait pratiquement $I_{\mathrm{e}} \simeq I_{\mathrm{i}}$ indépendant de $U_{\mathrm{p}}$ comme dans le grand caisson à parois isolantes.

Le nombre de collisions par électron dans un caisson à parois métalliques dépend de deux paramètres:

- la densité de plasma (courant d'ions émis par la source) comme dans l'expérience en caisson à parois isolantes ;

- le temps de confinement $\tau_{\mathrm{e}}$ fonction de $U_{\mathrm{p}}$.

Le critère $\bar{v}_{\mathrm{e}} \tau_{\mathrm{e}}>1$ pour avoir une distribution maxwellienne et une température électronique basse s'est toujours vérifié à un facteur 2 près $\left(0,5<\bar{v}_{\mathrm{e}} \tau_{\mathrm{e}}<2\right)$ pour 7 valeurs différentes de $U_{\mathrm{p}}$ et $10 \mu \mathrm{A}<I_{\mathrm{i}}<1 \mathrm{~mA}$.

4.4 RÉGLAGE DE LA TEMPÉRATURE ÉLECTRONIQUE $T_{\imath}$. - Le courant de saturation du filament neutraliseur est normalement très supérieur à $I_{\mathrm{e}}$. La chute de potentiel due au chauffage direct du filament est égale à plusieurs volts. Seule une petite fraction du filament dont le potentiel est inférieur ou approximativement égal au potentiel plasma émet des électrons ; le potentiel plasma est répulsif pour les électrons émis par l'autre partie (la plus positive) du filament. (On constate effectivement que le potentiel plasma est très proche du potentiel de la borne négative du neutraliseur soit $\simeq U_{\mathrm{p}}$ ). Si la température du filament décroît, la longueur du segment émissif croît (pour une valeur donnée de $I_{\mathrm{e}}$ ) ainsi que la chute de potentiel le long de celui-ci; il en résulte une légère augmentation du potentiel plasma et de l'énergie moyenne des électrons injectés dans le plasma. Ces électrons se refroidissent selon le processus décrit précédemment mais la température résultante du plasma croît.

La figure 4 montre la variation de $T_{\mathrm{e}}$ et du potentiel flottant $U_{\mathrm{f}}$ de la sonde en fonction du courant de chauffage $I_{\mathrm{n}}$ du neutraliseur. On constate qu'en réduisant $I_{\mathrm{n}}, T_{\mathrm{e}}$ croît de 500 à $2000 \mathrm{~K}$ et que $U_{\mathrm{f}}$ augmente

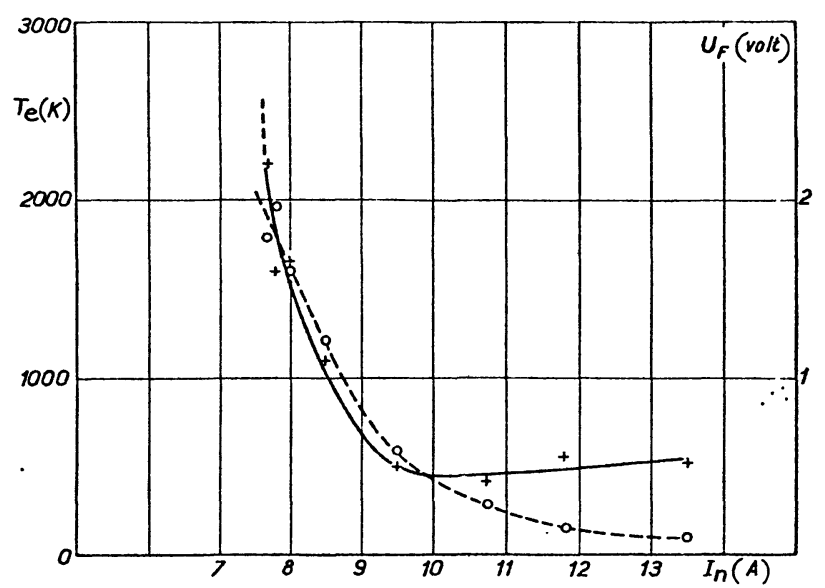

Fig. 4. - Température électronique $\left(T_{\mathrm{e}}\right)$ et potentiel flottant de la sonde $\left(U_{\mathrm{f}}\right)$ en fonction du courant de chauffage du neutrali$\operatorname{seur}\left(I_{\mathrm{n}}\right) .(+) T_{\mathrm{e}} ;(0) U_{\mathrm{f}}$.

de 2 volts. Pour $I_{\mathrm{n}}<7,5$ A (dans les conditions de la figure 4) le courant de saturation du filament devient inférieur au courant $I_{\mathrm{e}}$ nécessaire à la neutralisation du faisceau et celui-ci disparaît pratiquement comme dans le cas d'un filament froid $\left(I_{\mathrm{n}}=0\right)$.

5. Température ionique. $-\mathrm{La}$ mesure et le réglage de la température ionique ont fait l'objet d'une publication détaillée [13] ; un bref résumé est présenté ici.

Il convient de considérer deux composantes de la température ionique : la composante longitudinale $T_{\mathrm{i}_{\|}}$ (parallèle à la vitesse dirigée du faisceau) et la composante transversale $T_{i_{\perp}}$. Comme les fonctions de distribution peuvent être non maxwelliennes $T_{\mathbf{i}_{\|}}$et $T_{\mathrm{i}_{\perp}}$ sont définies comme des températures équivalentes déduites de l'énergie moyenne des ions.

La dispersion longitudinale de l'énergie des ions mesurée au moyen d'un analyseur électrostatique varie peu avec l'énergie moyenne et est de l'ordre de $\pm 2 \mathrm{eV}$. Pour la simulation du vol ionosphérique on doit considérer la température des ions dans un système de référence se déplaçant à la vitesse moyenne des ions. Dans ces conditions $T_{\mathrm{i}_{\|}}$est de l'ordre de 1500 à $2000 \mathrm{~K}$ ce qui convient parfaitement. Les collisions et l'action des champs électromagnétiques étant négligeables la dispersion transversale maximum de la vitesse des ions $\Delta V_{\perp}$ est égale à $V a / r$ où $a$ est le rayon de la grille d'extraction de la source d'ions. Dans les conditions de ces expériences $\Delta V_{\perp} / V \simeq 1 / 200$ ce qui correspond à $T_{\mathrm{i}_{\perp}} \simeq 6 \mathrm{~K}$. La théorie de l'interaction d'un corps avec un plasma non collisionnel et en mouvement montre que la composante de la température ionique à considérer est $T_{\mathrm{i}_{\perp}}$ (seul $\Delta V_{\perp}$ contribue au remplissage du sillage). Pour cette raison nous avons essayé d'accroître la valeur de $T_{\mathrm{i}_{\perp}}$.

Deux méthodes de mesure de $T_{\mathrm{i}_{\perp}}$ ont été employées :

- l'utilisation de l'effet de bout d'une sonde cylindrique longue [14], [15] ;

- un collimateur mis au point à l'ONERA. 
Le courant d'ions collecté par la sonde cylindrique longue ou par le collecteur du collimateur est fonction de l'angle entre la direction du faisceau et l'axe de la sonde ou du collimateur. Plus précisément le courant collecté passe par un maximum très aigu pour un angle nul ; la largeur à mi-hauteur du pic est de l'ordre de quelques degrés et croît avec $T_{\mathrm{i}_{\perp}}$; celle-ci a été calculée en fonction de $T_{\mathrm{i}_{\perp}}$ (en supposant une distribution maxwellienne) pour la sonde cylindrique longue [16] et pour le collimateur [17]. Les deux méthodes sont en assez bon accord [13].

La température transversale est ajustée au moyen d'une grille immergée dans le faisceau à environ $150 \mathrm{~cm}$ de la source d'ions (voir Fig. 1) et polarisée négativement. On constate que $T_{\mathrm{i}_{\perp}}$ crôt avec la tension de polarisation de la grille à condition que la grille et la densité du plasma soient telles que $a / h>3$ où $a$ est la dimension de la maille et $h$ la longueur de Debye au niveau de la grille. Pour $a / h<3$ la grille polarisée à des potentiels de plus en plus négatifs arrête les électrons (absence de neutralisation entraînant une baisse brutale de la densité d'ions en aval de la grille) avant qu'un effet notable sur $T_{\mathrm{i}_{\perp}}$ ne se soit produit.

L'interposition de la grille laissée au potentiel flottant introduit déjà une légère augmentation de $T_{\mathrm{i}_{\perp}}$ [18]. Une tension de polarisation de l'ordre de -20 volts permet d'obtenir $T_{\mathrm{i}}$ de l'ordre de 500 à $1000 \mathrm{~K}$. Une tension de polarisation encore plus élevée ( -40 volts) permet d'atteindre la condition $T_{\mathrm{i}_{\perp}} \simeq T_{\mathrm{e}}$ mais dans ce cas la fonction de distribution est non monotone.

Le réglage de $T_{\mathrm{i}}$ demeure très délicat et n'a été effectué que pour des études de sillage pour lesquelles la température ionique est un paramètre essentiel.

6. Perturbations dues aux molécules neutres. La pression résiduelle doit être assez basse pour que le plasma soit pratiquement sans collision. Cette condition est apparemment satisfaite assez largement à la pression normale d'utilisation de l'enceinte (source d'ions en fonctionnement) qui est de l'ordre de $10^{-4} \mathrm{~N} / \mathrm{m}^{2}$. En effet le libre parcours des ions argon de $20 \mathrm{eV}$ est égal à $100 \mathrm{~m}$ soit environ 16 fois la longueur de leur parcours dans l'enceinte. Pourtant l'analyse détaillée de la structure du sillage d'un corps immergé dans le plasma a montré que ce niveau de vide est en fait insuffisant.

Des ions froids sont produits par échange de charge entre les ions du faisceau et les molécules neutres résiduelles. Ces ions froids de vitesse très inférieure à celle du faisceau tendent à s'accumuler dans l'enceinte d'autant plus que les dimensions de celle-ci sont grandes. La conséquence la plus évidente de la présence d'ions froids est l'introduction d'une erreur dans la mesure de la densité d'ions du faisceau, plus particulièrement dans le sillage proche d'un corps où cette densité est théoriquement nulle ou très faible. Un effet plus subtil mais en pratique plus important est de perturber la distribution de potentiel du sillage proche (dominée par la charge d'espace électronique) et de modifier ainsi toute la structure du sillage (y compris le sillage lointain).

La figure 5 montre la structure du sillage (profils de densité d'ions) d'une sphère au potentiel flottant de $10 \mathrm{~cm}$ de diamètre, dans des conditions de plasma

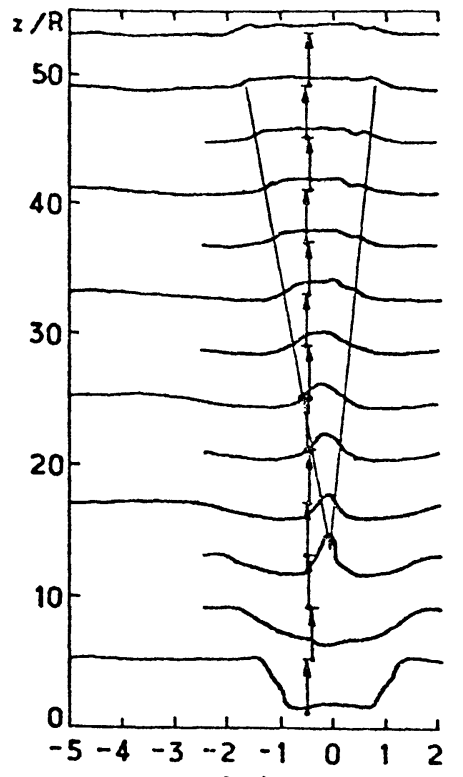

(o)

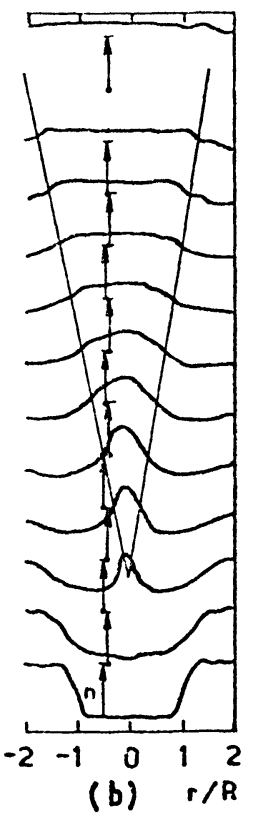

(b)
Fig. 5. - Sillage d'une sphère au potentiel flottant et de $10 \mathrm{~cm}$ de diamètre pour $R / h=22, T_{\mathrm{e}}=1600 \mathrm{~K}, E=20 \mathrm{eV}, T_{\mathrm{e}} / T_{\mathrm{i}} 10$, $M=15$ pour les pressions $p=2 \times 10^{-4} \mathrm{~N} / \mathrm{m}^{2}$ (a) et $1,5 \times 10^{-5} \mathrm{~N} / \mathrm{m}^{2}$ (b). La longueur des flèches indique la densité du plasma non perturbé ; leur origine donne la distance $z / R$ à laquelle le profil de densité a été mesuré.

en principe identiques mais à deux pressions différentes $2 \times 10^{-4} \mathrm{~N} / \mathrm{m}^{2}$ (Fig. $5 a$ ) et $1,5 \times 10^{-5} \mathrm{~N} / \mathrm{m}^{2}$ (Fig. $5 b$ ). Les paramètres $z / R$ et $x / R$ sont respectivement les distances longitudinales et transversales normalisées au rayon $R$ de la sphère.

A la pression la plus élevée, la présence d'une quantité d'ions froids très modérée (environ $5 \%$ de la densité d'ions en faisceau) a des effets importants sur la structure du sillage :

- accroissement de la densité apparente dans le sillage proche ;

- atténuation du contraste entre les régions de condensation et de raréfaction de la densité ;

- accroissement de la distance à laquelle un pic de densité apparaît ;

- décroissance de l'angle de propagation de la région de condensation de la densité.

Toutefois aucun changement de structure du sillage n'est observé lorsque la pression varie entre $1,5 \times 10^{-5} \mathrm{~N} / \mathrm{m}^{2}$ et $4 \times 10^{-5} \mathrm{~N} / \mathrm{m}^{2}$. On peut en conclure que l'effet des ions froids est négligeable aux pressions les plus basses. La pression minimum requise $\left(4 \times 10^{-5} \mathrm{~N} / \mathrm{m}^{2}\right)$ pour une étude quantitative du sillage est d'un ordre de grandeur inférieur à ce qui 
était obtenu et généralement considéré comme suffisant dans toutes les expériences antérieures [4], [5], [6], [7], [13]. Les pressions les plus basses ont été obtenues au moyen d'un piège à hélium liquide placé aussi près que possible de la source d'ions mais sans perturber le faisceau (Fig. 1). Ce piège consomme plus de 501 d'hélium liquide à l'heure mais son emploi ne se justifie que pour l'étude quantitative du sillage.

Récemment, une étude de l'influence des ions froids sur le profil de densité électronique et sur l'accroissement de la température électronique dans le sillage proche a confirmé l'importance de ce phénomène et la nécessité d'une pression résiduelle très basse [19].

7. Exemples d'utilisation. - Cette installation a été utilisée pour des essais d'expériences spatiales conduites par plusieurs laboratoires, par exemple [8]. Ces expériences comportaient: sondes de Langmuir, analyseurs électrostatiques, senseurs directionnels d'ions, sondes quadrupolaires, sondes à impédance, spectrographe de masse et détecteurs d'ultra-violet.

La structure du sillage de corps sphériques et cylindriques a également été étudiée et un bon accord avec les théories existantes a été obtenu [18]. A titre d'exemple la figure 6 montre la structure d'onde

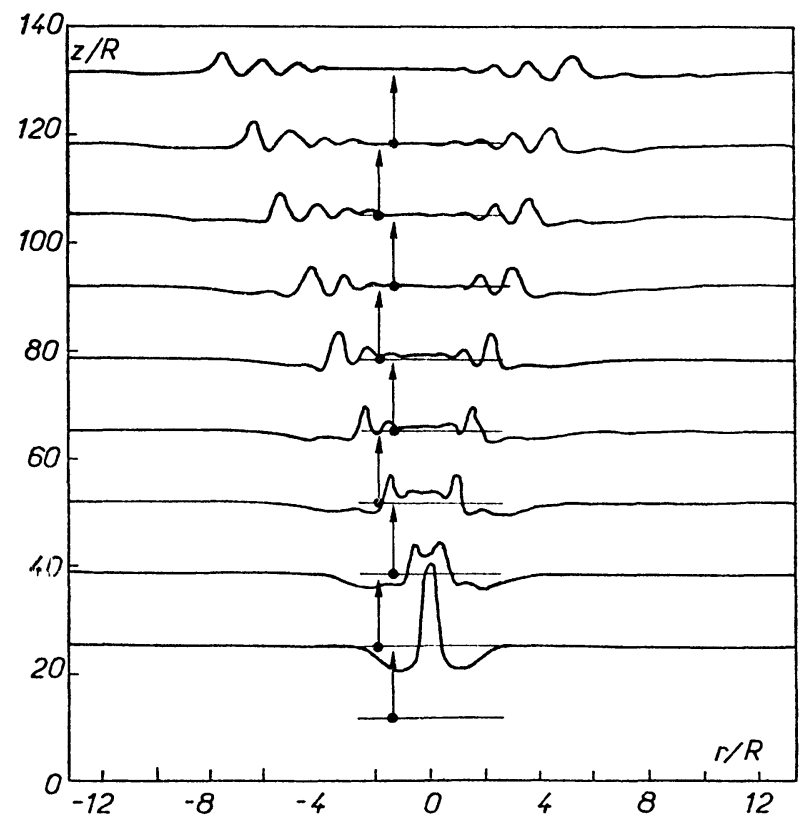

FIG. 6. - Sillage d'une sphère au potentiel flottant et de $3 \mathrm{~cm}$ de diamètre pour $R / h=6, T_{\mathrm{e}}=2000 \mathrm{~K}, E=20 \mathrm{eV}, T_{\mathrm{e}} / T_{\mathrm{i}}=100$, $M=15$ et une pression $p=1,5 \times 10^{-5} \mathrm{~N} / \mathrm{m}^{2}$.

ionique observée dans le sillage d'une sphère au potentiel flottant et de $30 \mathrm{~mm}$ de diamètre dans un plasma à température ionique très faible $\left(T_{\mathrm{e}} / T_{1} \simeq 100\right)$, sans effet d'ions froids (pression $1,5 \times 10^{-5} \mathrm{~N} / \mathrm{m}^{2}$ ) et pour un nombre de Mach électronique $M=15$. La figure 7 montre le sillage de la même sphère dans les mêmes conditions sauf en ce qui concerne la température ionique : $T_{\mathrm{e}} / T_{\mathrm{i}}=0,7$ et $M=8$; la variation $\mathrm{du}$

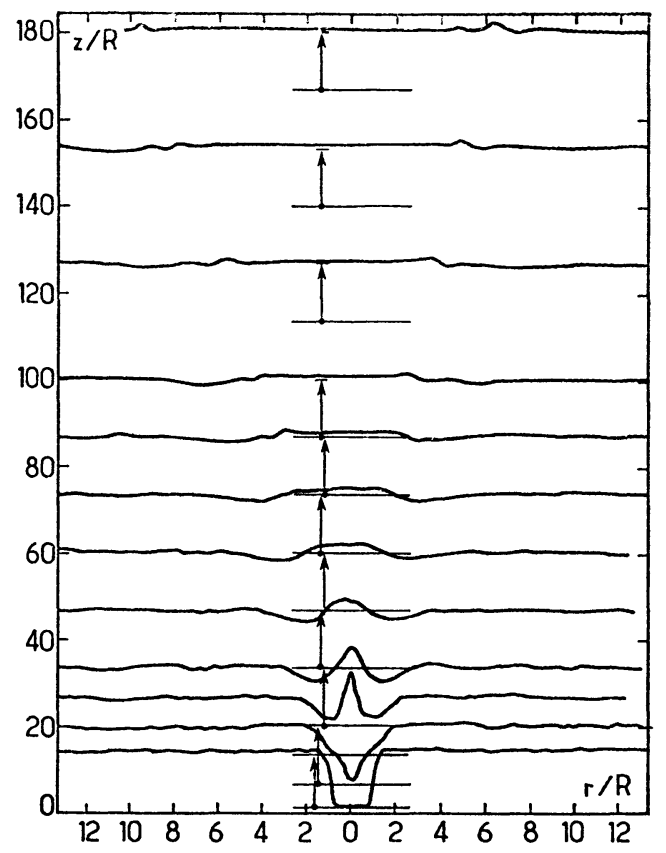

FIG. 7. - Sillage d'une sphère au potentiel flottant et de $3 \mathrm{~cm}$ de diamètre pour $R / h=7, T_{\mathrm{e}}=1400 \mathrm{~K}, E=20 \mathrm{eV}, T_{\mathrm{e}} / T_{\mathrm{i}}=0,7$, $M=8$ et une pression $p=1,5 \times 10^{-5} \mathrm{~N} / \mathrm{m}^{2}$.

nombre de Mach par rapport au cas précédent est due à l'influence de la température ionique

$$
M=V\left[k T_{\mathrm{e}}\left(1+3 T_{\mathrm{i}} / T_{\mathrm{e}}\right) / m\right]^{-1 / 2} .
$$

On remarque qu'une température ionique proche des conditions effectivement rencontrées dans l'ionosphère c'est-à-dire $T_{\mathrm{i}} \simeq T_{\mathrm{c}}$ a pour effet d'amortir complètement toute structure d'onde ainsi que le pic de densité dû à la focalisation des ions derrière la sphère. Les nombreuses études théoriques et expérimentales antérieures faites avec la condition $T_{\mathrm{i}} \ll T_{\mathrm{e}}$ ont conduit à des résultats intéressants d'un point de vue fondamental mais sans grand intérêt pratique pour la connaissance des conditions de vol ionosphérique.

8. Conclusion. - Cette installation permet de simuler les conditions du vol ionosphérique. Le domaine de variation des paramètres densité de plasma, température électronique et nombre de Mach électronique couvre pratiquement toutes les conditions susceptibles d'être rencontrées dans l'ionosphère. Ces trois paramètres peuvent être ajustés aisément et indépendamment les uns des autres dans des conditions stables et reproductibles.

L'étude quantitative du sillage ionosphérique a été effectuée mais a nécessité un niveau de vide plus poussé et l'ajustement d'un paramètre supplémentaire : la température des ions. La mesure de cette température est délicate et son réglage demeure très difficile à réaliser.

Les limitations de cette installation sont les suivantes :

- la densité maximum obtenue, compte tenu de la 
divergence du faisceau, ne permet pas l'étude quantitative du sillage de gros objets $(R / h>10)$;

- les variations de la densité de plasma sur une distance égale à $1 \mathrm{~m}$ sont de l'ordre de $10 \%$; ces variations sont gênantes pour la mise au point des sondes $\mathrm{HF}$ à vocation spatiale dont le volume d'interaction avec le plasma est de l'ordre d'un mètre cube ou plus ;

- une température électronique supérieure à $2000 \mathrm{~K}$ (avec une distribution maxwellienne) ne peut être obtenue ; avec une distribution non maxwellienne la température ne dépasse pas 3000 à $4000 \mathrm{~K}$;

- le nombre de Mach électronique minimum est de l'ordre de 8 ;

- les effets (d'ailleurs mineurs) du champ magnétique terrestre sur le sillage ne sont pas simulés ; toutefois un champ magnétique variable entre 0,1 et 1 gauss permet l'étude des effets liés à la gyrofréquence électronique (pour les sondes HF).

\section{Bibliographie}

[1] Sellen, Jr, J. M., Bernstein, W., Kemp, R. F., Rev. Sci. Instrum. 36 (1965) 316.

[2] Bonnal, J. F., Chavy, Y., Manus, C., Spiess, G., Conference on Physics of Quiescent Plasma, Frascati (1967).

[3] SpIEss, G., Thèse Paris-Orsay (1968).

[4] Hall, D. F., Kemp, R. F., Sellen Jr, J. M., AIAA J 3 (1965) 1490.

[5] Hester, S. D., Sonin, A. A., AIA A J 8 (1970) 1090.

[6] Oran, W. A., Stone, N. H., SAMIr, U., Planet. Space Sci. 22 (1974) 379.

[7] Pigache, D., aIAA J 11 (1973) 129.

[8] Groupement de Recherches Ionosphériques, Orléans (France) et Institut für Physikalische Weltraumforschung, Freiburg (R. F. A.)
[9] Le Vaguerese, P., Pigache, D., Revue Phys. Appl. 6 (1971) 325 .

[10] Charpenel, M., Pigache, D., N-T ONERA nº 202 (1972).

[11] SPITZER, L., Physics of fully ionized plasma (John Wiley et Sons, Inc) 1962.

[12] Le Vaguerese, P., Thèse Paris-Orsay (1970).

[13] Fournier, G., Pigache, D., J. Appl. Phys. 43 (1972) 4548.

[14] Bettinger, R. T., Chen, A. A., J. Geophys. Res. 73 (1968) 2513.

[15] Hester, S. D., Sonin, A. A., Phys. Fluids 13 (1970) 1265.

[16] Sammartin, J. R., Phys. Fluids 15 (1972) 1134.

[17] Fournier, G., J. Geophys. Res. 76 (1971) 3159.

[18] Fournier, G., Pigache, D., Phys. Fluids 18 (1975) 1443.

[19] Oran, W. A., Samir U., Stone, N. H., AIAA J 14 (1976) 1141. 